\title{
L'esperienza di un sito web per la dieta nelle malattie renali
}

\author{
Franca Pasticci ${ }^{1}$, Antonio Selvi ${ }^{2}$ \\ ${ }^{1}$ Dietista, U.O.C. Nefrologia e Dialisi, USL 1 dell'Umbria, Todi (PG) \\ ${ }^{2}$ Nefrologo, Direttore U.O.C. Nefrologia e Dialisi, USL 1 dell'Umbria, Assisi
}

\begin{abstract}
A WEBSITE FOR DIET IN RENAL DISEASE: OUR EXPERIENCE
Abstract. Introduction: Raising awareness of Chronic Kidney Disease (CKD) causes and disseminate nutritional knowledge may contrast the chronic and progressive course of the disease. Is becoming more and more common to find answers in the Web to the limitations imposed by the disease.

Methods: Websites dedicated to the CKD were examined and, on the basis of national and international experiences, was launched a website dedicated to nutrition in nephrology.

Results: One year after the site launch, visits are about 20 in daily averages, with peaks up to 60-140 people in relation to the insertion of new pages. Visitors are mostly people with CKD in conservative management or who were transplanted.

Conclusions: In the treatment of CKD a central profession is the renal dietitian. To date, there are few Dietitians available in nephrology centers; this website, as well as providing nutritional information, may increase the sensitivity of public opinion and institutions towards this professional figure.
\end{abstract}

Key words: Web, Nutrition, Renal disease

Conflict of interest: None.

Financial support: None.

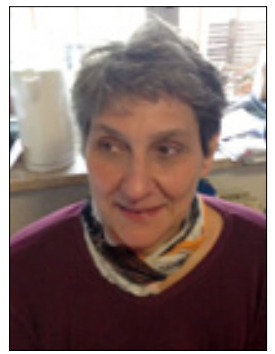

Franca Pasticci

\section{Introduzione}

La malattia renale cronica (MRC) è diventata ormai un problema di salute pubblica. È stato, infatti, stimato che ne sia affetto 1 individuo ogni 10 della popolazione generale adulta.

Visto l'andamento cronico della malattia e i suoi costi individuali e sociali, il suo trattamento dovrebbe essere una delle priorità del Servizio Sanitario, verso cui indirizzare gli sforzi di prevenzione.

In altri termini, sarebbe auspicabile che, almeno nei soggetti a rischio di MRC, venissero fatti sforzi volti a identificare precocemente i segnali di disfunzione per intervenire sulle cause ed evitare l'inizio della malattia o rallentarne il carattere evolutivo e, nelle fasi avanzate, volti a contrastare le devastanti complicazioni che si aggiungono alla malattia renale una volta che si è instaurata.

Oltre alle cause ereditarie, fattori di rischio per la comparsa di insufficienza renale cronica (IRC) sono quelli comuni alle malattie cardiovascolari: obesità, diabete, ipertensione, malattie cardiache, fumo ed età elevata. Esami semplici ed economici, come quello delle urine e la misura della pressione arteriosa, sono in grado di evidenziare alterazioni della funzione renale ben prima della comparsa dei sintomi.

Gli interventi di prevenzione primaria dovrebbero, quindi, essere rivolti a questi gruppi di popolazione, ed è riconosciuto che il trattamento dietetico è parte del trattamento medico di queste patologie. Infatti, l'intervento nutrizionale è fondamentale per ridurre i fattori di rischio e per contrastare l'aggravamento e/o l'instaurarsi di queste patologie.

La diffusione delle conoscenze e la sensibilizzazione al problema sono state riconosciute dalla Società Italiana di Nefrologia (SIN) e dalla Fondazione Italiana del Rene (FIR) come metodi utili per la prevenzione delle nefropatie e delle loro complicanze.

Accanto a specifiche campagne di informazione, in particolare in occasione della Giornata Mondiale del Rene (GMR), sono state dedicate a questo scopo aree dei siti istituzionali delle due società. In questo articolo, propongo un nuovo e personale sito web per dare alle persone strumenti utili per mettere in pratica $\mathrm{i}$ fondamenti del trattamento dietetico nel corso della nefropatia cronica e per contribuire, così, anche alla diffusione on-line dell'educazione nutrizionale nell'ambito delle malattie renali. 


\section{Materiali e metodi}

Per sviluppare il sito ho cercato sul web altri siti che trattassero lo stesso argomento. In Italia, ci sono diversi siti riguardanti le malattie renali.

Primo fra tutti, è da ricordare il sito della Fondazione Italiana del Rene:

http://www.fondazioneitalianadelrene.org/. La FIR è un'associazione Onlus che raccoglie i pazienti con patologia renale e tutti coloro, persone, associazioni e organizzazioni, che sono impegnati nell'affrontare le malattie dei reni per curarle, per limitarne $i$ danni o per prevenirle. Il sito della FIR si rivolge, quindi, a un'ampia platea, allo scopo di divulgare la conoscenza e la prevenzione della malattia renale. Rispetto alla dieta, la trattazione è scarna ed è rivolta sia ai pazienti in terapia conservativa che a quelli in terapia sostitutiva tramite dialisi. Al momento, nel sito della SIN, non vi sono notizie riguardanti la dieta nel corso di malattie renali. Recentemente, la stessa SIN, in collaborazione con la filiale italiana dell' Associazione Europea degli Infermieri di Dialisi e Trapianto/Associazione Europea per il Trattamento delle Malattie Renali EDTNA/ERCA e con 1'Associazione Nazionale Emodializzati, Dialisi e Trapianto ANED, ha promosso l'apertura di un nuovo spazio web, con l'obiettivo di fornire ai cittadini informazioni "qualificate e certificate" su prevenzione, diagnosi e cura delle malattie renali. Il sito www.curaituoireni.it mette a disposizione materiale utile per un'informazione e una gestione assistenziale corrette dei pazienti; poco si trova per quanto riguarda la dieta.

Un altro sito http://www.renalgate.it/, curato dal nefrologo Dr. Giuseppe Quintaliani, nella homepage si presenta come: il portale di tutto quello che riguarda le malattie renali e il loro trattamento sia strumentale che sostitutivo che palliativo. Cercheremo di inserire materiale nostro ma anche di cercare quanto di meglio si trova nel web sottoponendolo ai nostri esperti e filtrandolo per chi è meno esperto. Vogliamo essere, prima di tutto, un aiuto per i pazienti che si trovano in difficoltà, che non sanno a chi rivolgersi e che non hanno gli strumenti adeguati di conoscenza sia della malattia che dei meandri della sanità italiana... La nutrizione è il cavallo di battaglia del portale renalgate grazie all'esperienza e alla professionalità dei suoi collaboratori. Il sito si avvale di un comitato editoriale formato da professionisti delle diverse discipline. In questo sito, si trovano diverse presentazioni riguardanti la dieta e rivolte al team, come pure suggerimenti per i pazienti per la realizzazione della dieta sia in terapia conservativa che in terapia sostitutiva. Alcune pagine sono state realizzate con il mio contributo.

Altri siti che ho visitato sono quelli delle ditte che producono alimenti ipoproteici. Il più ricco è sicuramente quello della Aproten: http://www.aproten.it/. In questo sito, ci sono molte e ricche informazioni riguardo al trattamento nutrizionale dell'IRC in fase conservativa e il sito si sta arricchendo con una sezione curata da un cuoco e da una dietista che danno indicazioni rispetto alle tecniche di cottura idonee per gli alimenti ipoproteici e risposte alle domande dei pazienti.

Infine, per quanto riguarda il repertorio italiano, ho trovato il sito curato dalla Baxter, ditta farmaceutica. È un sito interna- zionale, in diverse lingue. Il sito italiano è:

http://italia.inforenal.com/italia/. Nella pagina iniziale, si legge che: Inforenal è un sito disegnato appositamente per le persone affette da insufficienza renale e per i loro familiari, amici e curanti. Offre informazioni di base sulla malattia renale, cause, sintomi e analisi, e sui diversi tipi di trattamento possibili.

Inoltre, Inforenal è stato approvato dalla SIN, è raccomandato dall'EDTNA/ERCA e ha il patrocinio dell'Associazione Italiana Donatori d'Organo (AIDO) e dell'Associazione Malati di Reni. È un sito abbastanza datato, ultimi aggiornamenti nel 2003, e non viene affrontata la dieta.

La versione in lingua inglese http://www.renalinfo.com/ è più aggiornata e ci sono accenni alla dieta come anche un elenco di ricette dedicate alle persone in dialisi.

Infine, nei siti delle associazioni dei pazienti, si trova la normativa di interesse ma non si trova nulla riguardo alla dieta.

Molto più vario e interessante è il panorama che si ha nei siti esteri, in particolare in lingua inglese. Il più famoso e ricco di informazioni è quello della National Kidney Foundation (NKF), dove c'è una sezione interamente dedicata ai pazienti e, all'interno di questa, una parte dedicata alla dieta nella malattia renale. Il sito http://www.kidney.org/ è, quindi, particolarmente ricco di spunti e suggerimenti per i pazienti.

Il sito http://www.worldkidneyday.org/ è il sito dedicato alla pubblicità della giornata mondiale del rene, dove è possibile reperire molte informazioni sulla malattia renale e sul suo trattamento. Poco è presente riguardo alla dieta.

Infine, navigando sul web, ho trovato un sito che mi è piaciuto in maniera particolare. Si tratta di http://www.kidneypatientguide.org.uk/contents.php, sviluppato grazie a un grant da una serie di professionisti della salute, esperti in tecnologia e media, di scrittori e di persone con malattia renale e dai loro familiari.

Nella homepage si legge: questo sito web fornisce informazioni per pazienti nefropatici, per i loro partner e le loro famiglie, per gli operatori sanitari e per chiunque altro sia interessato alla malattia renale. Si tratta di informazioni non solo sugli aspetti fisici dell'insufficienza renale, come la funzione renale o che cosa accade quando non funziona il rene, e sulle terapie disponibili, ma anche su questioni più ampie, come le implicazioni emotive, sociali e finanziarie.

Il sito è stato progettato in primo luogo per il Regno Unito, ma sarà utile per chi è affetto dalla malattia. Ci auguriamo che i visitatori troveranno un'utile fonte di informazioni e di supporto e che questo sito sarà l'occasione, per i pazienti nefropatici, gli accompagnatori e i familiari, per condividere le loro esperienze e i loro punti di vista. Il sito è ancora in fase di sviluppo, quindi, se volete darci un feedback o farci avere idee e suggerimenti, si prega di inviare un messaggio sul nostro forum.

A parere della sottoscritta è il sito che maggiormente si avvicina alle necessità dei pazienti, in quanto sono spiegati in modo semplice il funzionamento dei reni e le malattie renali, a volte con animazioni che aiutano molto nella comprensione.

C'è anche un buon accenno alla dieta anche se, come spesso accade, si tratta della dieta in terapia sostitutiva. 


\section{Risultati}

$\mathrm{Su}$ queste premesse è nato www.francapasticci.it, un sito per la dieta nelle malattie renali, curato da una dietista. Il sito è indirizzato ai pazienti, con la finalità di offrire loro un aiuto pratico, gratuito e semplice e, in questo modo, di dare forza all'approccio dietetico-nutrizionale per la prevenzione e la cura delle nefropatie croniche.

In questo primo momento, è stato inserito il materiale da noi prodotto negli anni e indirizzato ai pazienti.

Una sezione è dedicata alla valutazione bromatologica di piatti consumati usualmente sulle nostre tavole. La pasta al pomodoro, gli involtini di vitello, l'insalata, la mela, ... avranno visualizzati i valori di acqua, proteine, grassi e carboidrati. È indicato il valore assoluto, ma, accanto a questo, abbiamo scelto di mettere un diagramma a torta che mostra graficamente la composizione del piatto: ciò permette di rendere visibile che, con una pera di $100 \mathrm{~g}$, si introducono $85 \mathrm{~g}$ di acqua, $0.3 \mathrm{~g}$ di proteine, $0.4 \mathrm{~g}$ di grassi e $9.5 \mathrm{~g}$ di carboidrati. In altre parole, è stata inserita anche l'acqua fornita dall'alimento visto che, nella dieta, $\mathrm{e}$, in particolare, in quella in dialisi, sono da conteggiare anche i liquidi introdotti con l'alimentazione. Questo è un problema importante per i pazienti che si trovano a dover centellinare le bevande. In questo modo speriamo che sia possibile capire che non solo il cocomero contiene acqua ma che anche la pera si beve.

In relazione al controllo dell'apporto di fosforo e potassio, sono stati realizzati due diagrammi a torta, rispettivamente per potassio e fosforo. Nei diagrammi viene visualizzato quanto potassio (e fosforo) quell'alimento apporta in percentuale rispetto all'apporto giornaliero suggerito per un paziente con malattia renale e trattato con emodialisi. Nel caso della pera, questa fornisce circa il 5.5\% dell'apporto giornaliero di potassio consigliato e circa 1' $1 \%$ dell'apporto di fosforo consigliato. Accanto ai diagrammi viene riportato anche e sempre il valore assoluto.

Secondo le indicazioni delle Linee Guida internazionali sono suggeriti, per un individuo di $65-70 \mathrm{~kg}$, di 60 anni d'età, con malattia renale e trattato con emodialisi, i seguenti apporti:

- energia: circa 2100 calorie (30-35 Kcalorie per kg di peso corporeo)

- proteine: circa 70-80 g (1-1.2 g per kg di peso corporeo)

- fosforo: circa $1000 \mathrm{mg}$ al giorno

- potassio: inferiore a $2400 \mathrm{mg}$ al giorno.

L'elaborazione bromatologica è fatta con i dati forniti dalla Banca Dati di composizione degli alimenti per studi epidemiologici in Italia, Istituto Europeo di Oncologia, Edizione 2002. La sezione delle ricette è una sezione interattiva: infatti, è prevista la possibilità di invio di ricette da valutare nella composizione . Le ricette sono poi pubblicate sul sito con la relativa valutazione bromatologica e con note appropriate. È prevista anche la possibilità di inviare domande da poter mettere in comune tra $\mathrm{i}$ fruitori del sito in una sezione FAQ.

\section{Discussione}

Il sito, messo sul web a metà Dicembre del 2012, è stato pubblicizzato tramite conoscenze personali e gruppi Facebook dei quali sono entrata a far parte.

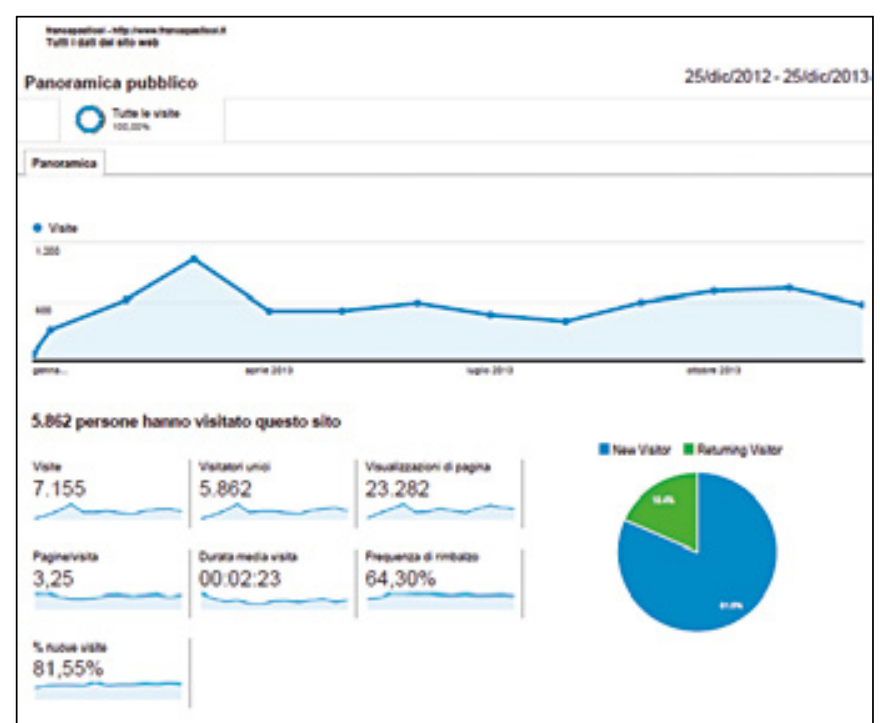

Fig. 1 - Visite al sito: dati da Google Analytics nel periodo 25/12/2012 25/12/2013.

In un anno dal lancio (25/12/2012-25/12/2013) le visite sono state 5862 (dati ricavati da http://www.google.it/intl/it/ analytics/), con circa 20 visitatori al giorno e punte di 60-140 persone. Il $18.4 \%$ è costituito da visitatori che tornano; la durata media delle visite è di 2.23 minuti. I visitatori che ritornano, di solito, stanno più tempo sul sito, con una media di 5.30 minuti (Fig. 1).

Le pagine più visitate sono quelle delle ricette e quelle relative alla dieta in terapia conservativa.

I picchi registrati sono in relazione all'inserimento di notizie, pagine e ricette, poi pubblicizzate tramite Facebook. Questo social network è la sorgente numericamente più consistente di accessi.

I contatti dal sito sono stati circa 20; sono persone che hanno fatto richieste personali e non domande che è stato possibile condividere nella sezione FAQ. È stato chiesto soprattutto uno schema dietetico; tali richieste non sono state soddisfatte, visto che la terapia dietetica è legata allo stato clinico ed è una terapia da adattare agli obiettivi, alle priorità, alla capacità, alla cultura e allo stile di vita dell'individuo.

Alcuni pazienti hanno inviato piatti realizzati da loro chiedendone la visualizzazione bromatologica. Sono ricette per la terapia conservativa realizzate con alimenti ipoproteici, e ciò ha richiesto l'apertura di una sezione ad hoc.

I visitatori sono, per la maggior parte, persone con insufficienza renale cronica in terapia conservativa o che hanno fatto il trapianto; pochi sono i contatti da individui in dialisi, sebbene il sito sia stato espressamente dedicato a costoro. È possibile ipotizzare che la maggior parte delle persone in dialisi sia anziana e abbia poca dimestichezza con il web?

Limiti del progetto sono:

- la necessità di un continuo aggiornamento che contrasta con i tempi a disposizione del webmaster;

- lo sviluppo nella sola lingua italiana che non permette l'utilizzo del sito da parte di utenti di nazionalità diverse. 


\section{Conclusioni}

Le malattie renali modificano la qualità di vita dei pazienti in quanto essi devono mettere in pratica cambiamenti importanti e difficili dello stile di vita, compresi quelli delle abitudini alimentari. Di contro, è riconosciuto a livello mondiale che comportamenti dietetici corretti possono aiutare a controllare i fattori di rischio delle nefropatie e contribuire a prevenirle e a rallentarne l'evoluzione e le complicanze; la conoscenza dovrebbe, inoltre, migliorare l'adesione alle limitazioni imposte e permettere, nel contempo, di fare scelte in base alle proprie preferenze.

Per le malattie croniche, come il diabete o le malattie renali, è necessario, infatti, che siano programmate strategie di intervento che consentano ai pazienti di prendere decisioni responsabili e di mettere in atto comportamenti di auto-cura, assumendosi la propria responsabilità per il quotidiano.

Nelle nefropatie, la figura del dietista e, in particolare, del dietista renale è centrale, in quanto è l'operatore sanitario in grado di integrare i risultati della storia medica, della storia dietetica e della valutazione nutrizionale per sviluppare un piano dietetico personalizzato sulla base delle attitudini e delle preferenze del paziente. Purtroppo, a tutt'oggi, pochi sono i dietisti disponibili nelle strutture di nefrologia e il sito può aumentare la sensibilità dell'opinione pubblica e, speriamo, delle istituzioni.

Il sito ha necessità di un continuo aggiornamento ed è aperto al contributo di tutti gli operatori coinvolti nel trattamento dei pazienti con malattia renale. Nella nostra idea, il sito potrebbe essere una piattaforma di collaborazione con nefrologi, infermieri e pazienti che vogliano pubblicare del materiale a beneficio dei visitatori.

\section{Riassunto}

Introduzione. La sensibilizzazione alle cause della malattia renale cronica e la diffusione delle conoscenze nutrizionali possono contrastare l'andamento cronico e progressivo della stessa. L'utilizzo del web è sempre più frequente come possibile mezzo di reperimento di risposte alle limitazioni imposte dalla malattia.

Metodi. Sono stati esaminati altri siti web dedicati alla MRC e, sulla base delle esperienze nazionali e internazionali, è stato creato un sito web dedicato alla nutrizione in nefrologia.

Risultati. A un anno di distanza dall'apertura del sito, le visite giornaliere medie sono circa 20 , con picchi fino a 60-140 persone in relazione con gli inserimenti di nuove pagine. I visitatori sono per la maggior parte persone con MRC in fase conservativa oppure che hanno fatto il trapianto.

Conclusioni. Nel trattamento delle nefropatie, la figura del dietista è centrale. A tutt'oggi, pochi sono i dietisti disponibili nei centri di nefrologia; il sito, oltre a fornire informazioni nutrizionali, potrebbe aumentare la sensibilità dell'opinione pubblica e delle istituzioni verso questo professionista.

Parole chiave: Web, Nutrizione, Malattia renale.

Dichiarazione di conflitto di interessi: Gli Autori dichiarano di non avere conflitto di interesse.

Contributi economici agli Autori: Gli Autori dichiarano di non aver ricevuto sponsorizzazioni economiche né per la realizzazione del sito né per la preparazione dell'articolo.

Questo articolo è stato presentato come prova finale del Master di I livello dell'Università di Pisa, "Nutrizione e Dietetica in Nefrologia" (Direttore Prof. Adamasco Cupisti).

Indirizzo degli Autori:

Dietista Franca Pasticci

USL 1 dell'Umbria

U.O.C. Nefrologia e Dialisi

Presidio Ospedaliero Media Valle del Tevere

Via del Buda

06059 Todi (PG)

emirocc@tin.it 